DE

M E D I C I N A

T R O P I C A L

$\mathrm{DE}$

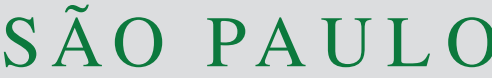

JOURNAL OF THE SÃO PAULO INSTITUTE OF TROPICAL MEDICINE

${ }^{1}$ Hospital General "Dr. Manuel Gea González", Departamento de Ecología de Agentes Patógenos, Ciudad de México, Mexico

${ }^{2}$ Hospital General "Dr. Manuel Gea González", Subdirección de Investigación, Ciudad de México, Mexico

${ }^{3}$ Hospital General "Dr. Manuel Gea González", Departamento de Biología Molecular e Histocompatibilidad, Hospital General "Dr. Manuel Gea González", Ciudad de México, Mexico

Correspondence to: Angélica Olivo-Díaz Hospital General "Dr. Manuel Gea González", Departamento de Biología Molecular e Histocompatibilidad, Calzada de Tlalpan 4800, Col. Sección XVI, Ciudad de México, 14080, Mexico

Tel: 52554000 3000, Ext. 6105

E-mail: aolivod@yahoo.com

Received: 15 May 2020

Accepted: 17 August 2020

\section{Interaction between human mucins and parasite glycoproteins: the role of lectins and glycosidases in colonization by intestinal protozoa}

\author{
Joel Martínez-Ocaña ${ }^{1}$, Pablo Maravilla ${ }^{{ }_{2}^{2}}$, Angélica Olivo-Díaz ${ }^{\oplus_{3}}$
}

\section{ABSTRACT}

Intestinal mucins are the first line of defense against microorganisms. Although knowledge about the mechanisms involved in the establishment of intestinal protozoa is limited, there is evidence that these parasites produce lectin-like molecules and glycosidases, that exert both, constitutive and secretory functions, promoting the establishment of these microorganisms. In the present review, we analyse the main interactions between mucins of the host intestine and the four main protozoan parasites in humans and their implications in intestinal colonization. There are lectin-like molecules that contain complex oligosaccharide structures and $\mathrm{N}$-acetylglucosamine (GlcNAc), mannose and sialic acid as main components, which are excreted/secreted by Giardia intestinalis, and recognized by the host using mannose-binding lectins (MBL). Entamoeba histolytica and Cryptosporidium spp. express the lectin galactose/ $\mathrm{N}$-acetyl-D-galactosamine, which facilitates their adhesion to cells. In Cryptosporidium, the glycoproteins gp30, gp40/15 and gp900 and the glycoprotein lectin CpClec are involved in protozoan adhesion to intestinal cells, forming an adhesion-attack complex. G. intestinalis and E. histolytica can also produce glycosidases such as $\beta$-N-acetyl-D-glucosaminidase, $\alpha$-d-glucosidase, $\beta$-d-galactosidase, $\beta$-l-fucosidase, $\alpha$-N-acetyl-d-galactosaminidase and $\beta$-mannosidase. In Blastocystis, $\alpha$-D-mannose, $\alpha$-D-glucose, GlcNAc, $\alpha$-D-fucose, chitin and sialic acid that have been identified on their surface. Fucosidases, hexosaminidases and polygalacturonases, which may be involved in the mucin degradation process, have also been described in the Blastocystis secretoma. Similarly, symbiotic coexistence with the intestinal microbiota promotes the survival of parasites facilitating cell invasion and nutrients obtention. Furthermore, it is necessary to identify and characterize more glycosidases, which have been only partially described by in silico analyses of the parasite genome.

KEYWORDS: Mucins. Lectins. Glycosidases. Protozoa. Intestinal parasites.

\section{INTRODUCTION}

The normal human gastrointestinal tract is inhabited by a vast and diverse community of microorganisms, such as bacteria, yeasts and parasites, and it is thought that a well-balanced microbiota is important for normal digestion and maintenance of the intestinal ecosystem. The mucosal epithelial tissue, after exposure to the external environment, has developed multiple functions and defense mechanisms in response to their susceptibility to microorganisms. Among the functions of mucosal epithelium are the absorption of nutrients and the role of cofactors in the intestine; gas exchange in the respiratory system; transparency of the cornea and lubrication; and detection of chemical elements. On the other hand, mucous membranes also have close relationships with the innate and the adaptive 
immune systems, allowing a rapid response to threats to the mucous surface ${ }^{1}$. Therefore, mucosal epithelia throughout the body can be considered dynamic and vital entities forboth, the normal function of the body and its interaction with internal and external environments. Considering that the gastrointestinal tract is one of most exposed epithelia to environmental aggressors, it is important to study its interactions with different microorganisms. Thus, some intestinal parasites that cause diseases in humans can express different molecules that interact with the intestinal epithelium of the host, such as lectins, glycosidases and mucin-like proteins, which can favour the binding with and the invasion of host cells as well as the evasion of the immune response. In addition, parasitic enzymes can also facilitate infection by degrading the host mucus or by generating binding sites to host cells ${ }^{2}$.

The present review focuses on the interaction between some molecules, such as lectin, lectin-like, mucin-like and glycosidase molecules which are related to the attachment and colonization by Giardia intestinalis, Entamoeba histolytica, Cryptosporidium spp. and Blastocystis spp. as the main parasitic protozoa that affect the intestinal mucosa of the human host, highlighting the possible role that these molecules play in the pathogenicity of these microorganisms.

\section{Mucins: the first line of defense of the intestinal epithelium}

The gastrointestinal mucosa is formed by a layer of columnar epithelial cells covered by a mucus coating. The mucus forms two layers, a sterile inner thin layer and a thicker non-sterile easy-to-remove layer. The viscous properties of mucus are generated by the secreted mucins, composed of large and complex glycoproteins bound to $O$-carbohydrates, representing approximately $70 \%$ of their composition and assembled as homo-oligomers ${ }^{3}$. In the gastrointestinal region, there are five secreted mucins that oligomerize (gel-forming) (MUC2, MUC5AC, MUC5B, MUC6 and MUC19) and a secreted mucin that does not oligomerize (non-gel-forming) (MUC7) (Table 1) 3.4. $^{\text {. Homo- }}$ oligomers are formed through intermolecular disulfide bonds between the cysteine-rich domains found at the amino and carboxyl ends of mucins. In the endoplasmic reticulum, $\mathrm{N}$-terminal glycosylation and C-terminal dimerization occur, and then, in the Golgi apparatus, $O$-glycosylation and N-terminal multimerization of the dimers continue, followed by the packing of the mucins into granules for subsequent secretion. The glycosyltransferases within the Golgi of the goblet cells determine the identity of the oligosaccharides that are secreted. The expression of these mucins can be modulated by the innate and the adaptive immunity and differs between sites in the gastrointestinal tract. In the stomach, the mucus is composed of MUC5AC and MUC6 and is produced by calyx cells. In the small intestine, the mucus layer is thinner (mainly composed of MUC2) and consists of an inner layer of $\sim 15-30 \mu \mathrm{m}$ and an outer layer of $\sim 100-400 \mu \mathrm{m}$, being thicker in the ileum. Mucus in the small intestine is produced by calyx cells and Paneth cells. In the large intestine, mucus (mainly MUC2) is predominantly produced by cells in the calyx and consists of an inner layer of $\sim 100 \mu \mathrm{m}$ and a thick outer layer of $\sim 100 \mu \mathrm{m}^{5}$.

Table 1 - Distribution of mucins in the gastrointestinal tract.

\begin{tabular}{lcc}
\hline Gastrointestinal Tract & Mucins & References \\
\hline Stomach & MUC1, MUC5AC, & MUC6 \\
& MUC1, MUC2, \\
Small intestine & MUC3, MUC4, & \\
& MUC6, MUC12, & $1,3-6$ \\
& MUC13, MUC17 \\
\hline \multirow{3}{*}{ Large intestine } & MUC1, MUC2, \\
& MUC3, MUC4, \\
& MUC12, MUC13, \\
& MUC17 \\
\hline
\end{tabular}

A mucin protein (apomucin) is composed of a tandem repeat arrangement with elevated proportions of proline, threonine and serine (PTS domain). Carbohydrates are bound to the PTS domains, constituting approximately $50 \%$ of the molecular weight and with sizes and numbers that vary according to the type of mucin. PTS domains are encoded by regions known as variable number of tandem repeats (VNTR), which are polymorphic and variable in different individuals and between distinct species ${ }^{6}$. Furthermore, the genes encoding glysosyl transferases are genetically variable, increasing intra- and inter-species diversity. This diversity is an important contributor to the specificity of commensal and pathogenic microorganisms, since the external composition of the mucins can have multiple ligands with which microorganisms can bind and can be used as energy sources ${ }^{3}$.

\section{Lectins, mucin-like molecules and glycosidases in intestinal parasites}

The definition of lectin is that it is a protein that has at least one non-catalytic domain that binds with a carbohydrate in a specific and reversible way ${ }^{7}$. In parasitic protozoa, lectins play important roles in different hostparasite interactions, as they facilitate the attachment to 
various glycoproteins, glycolipids and proteoglycans found on the surface of the host's gastrointestinal tract, which is the site of contact for invasion and colonization (Table 2) ${ }^{8}$.

Mucin-like glycoproteins have also been identified in several parasites, and these molecules are composed of amino acids and carbohydrates, which resemble mammalian mucins, in that they are highly glycosylated and rich in Thr, Ser and Pro. Proteophosphoglycans (PPGs) are the structural equivalents of mammalian mucins and have been detected in Entamoeba histolytica ${ }^{9}$, as well as in Cryptosporidium parvum ${ }^{10,11}$.

In addition, parasites that live in the mucosal epithelium require mechanisms to penetrate into the host's mucins, which can damage the mucous lining of the intestinal tract, as observed in Giardia, Entamoeba, and Trichomonas infections; these mechanisms may include the production of enzymes that degrade the protective epithelial mucus, the generation of ligands for the binding with and the invasion of the host's tissues and the release of nutrients that the parasite can consume ${ }^{2,12}$.

\section{Giardia intestinalis}

Giardia intestinalis colonizes the duodenum, jejunum, and ileum and is the etiological agent of human giardiasis, one of the most common gastrointestinal diseases worldwide. The clinical manifestations of giardiasis vary from no symptoms to acute or chronic diarrhoea with abdominal pain, flatulence, weight loss, intestinal lesions and malabsorption syndrome, which can last for several months. Giardia intestinalis exhibits two stages, the cyst (resistant form) and the trophozoite (the parasitic form). Giardia cysts have a hard wall and are impervious to small molecules, which protect them from the environment, but they can break through quickly when the parasite excysts and colonizes the duodenum ${ }^{13}$. The Giardia cyst wall contains $\beta$-1,3-linked $\mathrm{N}$-acetylgalactosamine (GalNAc), a homopolymer carbohydrate and at least three cyst wall proteins (CWPs) composed of Leu-rich repeats and a $\mathrm{C}$-terminal conserved Cys-rich region. The Leu-rich repeat domain of CWP1 is a lectin that binds with the curled fibrils of the GalNAc homopolymer ${ }^{14}$.

There is evidence of two main mechanisms that allow Giardia trophozoites to adhere to intestinal cells: a) a cytoskeletal mechanism involving ventral flagella or ventral discs, and b) a lectin-mediated mechanism ${ }^{15}$. Additionally, Giardia may produce $\alpha-\mathrm{N}$-acetyl-D-glucosaminidase, $\beta$-N-acetyl-D-glucosaminidase, $\alpha$-D-galactosidase, $\beta$-1-fucosidase, $\alpha$-mannosidase and $\beta$-xylosidase, enzymes that can degrade the intestinal mucins of the host, facilitating the invasion by the parasite ${ }^{16}$.

Alternatively, it has been described that MBL and ficolins (innate pattern recognition receptors [PRRs]) of the host are capable of recognizing GlcNAc on the surface of the parasite, activating the lectin-complement pathway, as demonstrated by the inhibition of this route with different concentrations of mannose in vitro ${ }^{17}$.

Furthermore, in the excretion/secretion products of $G$. intestinalis, two 58 and $63 \mathrm{kDa}$ glycoproteins were identified by the periodic acid-Schiff staining. However, when these products were treated with sodium metaperiodate or $\mathrm{N}$-glycosidase $\mathrm{F}$, a deglycosylation

Table 2 - Lectin or glycan-like structures and glycosidases secreted by the main protozoan parasites.

\begin{tabular}{|c|c|c|c|}
\hline Parasite & Lectin/glycan-like structures & Glycosidases & References \\
\hline Giardia lamblia & $\begin{array}{l}\text { Polymer b-1,3-linked GalNAc } \\
\text { specific in cyst, Secretion } \\
\text { N-glycan structures, including } \\
\text { GlcNAC, mannose and sialic } \\
\text { acid as main components }\end{array}$ & $\begin{array}{c}\beta \text {-N-acetyl-d-glucosaminidase, } \\
\alpha \text {-d-glucosidase, } \beta \text {-d-galactosidase, } \\
\beta \text {-I-fucosidase, } \alpha-N \text {-acetyl-d- } \\
\text { galactosaminidas, } \beta \text {-mannosidase }\end{array}$ & $12,14,16-18$ \\
\hline Entamoeba histolytica & Gal/GalNAc-specific surface & $\begin{array}{c}\beta \text {-N-acetyl-d-glucosaminidase, } \\
\alpha \text {-d-glucosidase, } \beta \text {-d-galactosidase, } \\
\beta \text {-l-fucosidase, } \\
\alpha-N \text {-acetyl-d-galactosaminidas, } \\
\beta-\text {-mannosidase, } \\
\alpha 1,6 \text {-glucosidase }\end{array}$ & $12,16,19-24$ \\
\hline Cryptosporidium parvum & $\begin{array}{c}\text { Gal/GalNAc-specific surface } \\
\text { (p30), gp15, gp40, gp900, } \\
\text { CpClec }\end{array}$ & INA & $37-46$ \\
\hline Blastocystis spp & $\begin{array}{l}\alpha-D-m a n n o s e, \alpha-D \text {-glucose, } \\
\text { GlcNAc, } \alpha \text {-L-fucose, chitin, } \\
\text { and sialic acid specific surface }\end{array}$ & $\begin{array}{c}\text { L-Fucosidase, Hexosaminidase, } \\
\text { Polygalacturonase, }\end{array}$ & $53-58$ \\
\hline
\end{tabular}

INA = information not available 
enzyme, serum from the mice immunized with excretion/ secretion products detected three proteins of 36,58 and $63 \mathrm{kDa}$, indicating the possibility of a glycosidic epitope recognition by antibodies ${ }^{18}$.

\section{Entamoeba histolytica}

Entamoeba histolytica is a mitochondria-devoided protist and the parasite responsible for human amebiasis; in the colon it may cause dysentery, but it can also spread through the blood circulation to other organs, mainly the liver, causing abscesses. The parasite has two life cycle forms: motile trophozoites and cyst forms. Trophozoites are the proliferative form crucial for the development of the disease. The cysts are the infective form, produced in the intestine and eliminated in the feces and subsequently ingested through contaminated food or water.

E. histolytica mainly expresses a Gal/GalNAc-specific surface lectin on its surface, which is associated with its virulence, thus facilitating adherence to the host's mucins and colonic cells. Certain virulent clones of E. histolytica can internalize and degrade the mucins of the host's colon and secrete them, facilitating the passage in to the mucosal epithelium through the protective mucus. Avirulent clones have a much lower rate of internalization and mucosal discharge ${ }^{12,19,20}$. Adhesion of trophozoites to colonic mucin is almost completely inhibited by the addition of Gal or GalNAc in vitro, although some attachment may still occur at high concentrations of these molecules, indicating the participation of other components in this interaction ${ }^{20,21}$. To lyse host epithelial cells, the parasite-host interaction through this lectin is necessary, as indicated by the addition of Gal or GalNAc inhibiting the general virulence of the parasite in vitro.

The Gal/GalNAc lectin is composed of a $260 \mathrm{kDa}$ heterodimer of heavy $(h g l)(170 \mathrm{kDa})$ and light $(l g l)$ $(35 / 31 \mathrm{kDa})$ subunits linked by disulfide bonds noncovalently associated with an intermediate subunit $(i g l)$ of $150 \mathrm{kDa}$. The $l g l$ and $i g l$ subunits are anchored to the membrane by acyl-glycosyl-phosphatidylinositol (GPI). The $h g l$ subunit has a carbohydrate recognition domain (CRD), within a cysteine-rich region (amino acids 356 to 1143), and has a short cytoplasmic tail, which is implicated in intracellular signalling and is homologous to the $\beta 2$ and $\beta 7$ integrins.

In E. histolytica, the $h g l$ subunit gene family consists of five genes with $89-95 \%$ of identity at the amino acid level. The $\mathrm{lgl}$ subunit is composed of a gene family of 6-7 members encoding isoforms, sharing 79-85\% identity, with different post-translational modifications. The $i g l$ subunit has two genes encoding proteins with $84 \%$ amino acid identity, but do not share similarity with the $260-\mathrm{kDa}$ protein $^{20,22}$.

The $l g l$ subunit does not appear to be involved in carbohydrate recognition, as mAb and polyclonal antibodies directed against the light subunit isoforms have no significant effect on adherence or cytotoxicity. When the $l g l$ protein is purified, it is always bound with the $h g l$ protein, however, when the $l g l$ protein lacks the GPI anchor, it does not bind with $h g l$, or with carbohydrates (Gal), indicating that $\mathrm{lgl}$, when not part of the heterodimeric lectin, lacks this capacity $^{23}$. Additionally, when amebas were transfected with a truncated $l g l$ gene, which encodes a protein produced in excess, there was a significant decrease in their ability to adhere and kill mammalian cells, to form rosettes with human red blood cells (which is mediated by the $\mathrm{Gal} / \mathrm{GalNAc}$ E. histolytica lectin incubated with red cells) and to phagocyte erythrocytes, and they were unable to cap anti-Gal lectin complexes on their surface (the process known as capping is the rapid redistribution of surface antigen-anti-body complexes in trophozoites), which indicates that the light subunit has the role of grouping these complexes, an activity that is essential for virulence ${ }^{24}$.

On the other hand, igl lacks a carbohydrate recognition motif; however, it has galactose-binding activity and contains several CXXC and CXC domains, which indicates protein-protein interactions; monoclonal antibodies against igl significantly inhibit ameba adherence, phagocytosis and toxicity ${ }^{25}$. In addition, $96 \%$ of hamsters immunized with the C-terminal fragment of $i g l$ were protected against liver abscess formation, and the antibodies generated inhibited up to $80 \%$ the adhesion of trophozoites to mammalian cells in vitro, supporting the use of this fragment as a vaccine to prevent amebiasis ${ }^{26}$.

Furthermore, the CRD of the Gal/GalNAc lectin has been shown to function as a pathogen-associated-molecularpattern-like molecule (PAMP), as it binds with Toll-like receptors TLR-2 and with TLR-4 on human colon cells, and it can induce the expression of elements of the innate immune response, subsequently leading to the production of pro-inflammatory cytokines. This is exacerbated when there is previous exposure to pathogenic enterobacteria, leading to the development of an invasive disease ${ }^{27}$. Another interesting activity described for Gal/GalNAc lectin is that the E. histolytica Igl1 subunit has both, hemolytic and cytotoxic activities and they depend directly on the level of expression of the $\mathrm{Lgll} \mathrm{gene}^{28}$.

The 220-kDa lectin (L220) has specificity for GlcNAc, but hemagglutination may be inhibited by hyaluronic acid, chitotriose, chitin, GalNAc and Gal. L220 was immunolocalized on the surface of the trophozoites, 
indicating their participation in adhesion to the target cells ${ }^{20}$.

The 112-kDa protein is also involved in adhesion and is located within the plasma membrane and into trophozoite vacuoles. The final protein is encoded by two nearby genes that give rise to a 49 and a $75 \mathrm{kDa}$ polypeptide. The $49-\mathrm{kDa}$ polypeptide is a cysteine protease (EhCP112), which can digest azocasein, gelatine, type I collagen, fibronectin and haemoglobin, while the 75-kDa polypeptide is an adhesin (EhADH); both polypeptides are located on the surface of trophozoite as they are membrane proteins. The activity of the cysteine protease allows mucin degradation and parasite access to the epithelium of the host colon, which causes increased mucin secretion to prevent invasion of the parasite. EhCP112 also has a cathepsin (Cys-His-Asn) catalytic domain and an RGD (Arg-Gly-Asp) domain, suggesting that this molecule can bind with integrins s $^{20,27,29}$.

Several glycosidases secreted by or found in the lysates of E. histolytica trophozoites have been described, including $\beta$-N-acetyl-D-glucosaminidase, $\alpha$-D-glucosidase, $\beta$-D-galactosidase, xylosidase, glucuronidase, $\mathrm{N}$-acetylneuraminate lyase, neuraminidase, hyaluronidase, $\beta$-L-fucosidase, $\alpha$-N-acetyl-galactosaminidase, sialidase, $\beta$-amylase and $\alpha$-mannosidase ${ }^{16,30-32}$. Of these, sialidase, $\alpha$-glucosidase, $\beta$-amylase and $\beta$-N-acetyl-Dglucosaminidase seem to be involved in ameba pathogenesis, being the most active $\beta$-N-acetyl-D-glucosaminidase, which seems to have a central role in the degradation of mucin $^{31}$. Glycosidases act through the degradation of mucin carbohydrates, thus exposing the protein skeleton to parasite proteases $^{31,33}$. Cysteine proteases secreted by E. histolytica are mainly involved in the final disruption of colonic mucin, allowing ameba adherence and invasion ${ }^{34}$.

\section{Cryptosporidium spp.}

Cryptosporidium is an obligate intracellular protozoan parasite, a member of the Apicomplexa phylum, which was initially recognized as an opportunistic pathogen in immunocompromised patients, but can also be found in immunocompetent hosts, usually causing asymptomatic or self-limited infections ${ }^{35}$. The species that mainly infect humans are C. hominis and C. parvum ${ }^{36}$. Cryptosporidium life cycle is complex, starting with the ingestion of food or water contaminated with oocysts, followed by an excystation process in the small intestine, going through various stages of asexual and sexual reproduction in the intestinal epithelial cells, to finally produce oocysst that are going to be released into the intestine and excreted into the environment ${ }^{37}$.

The genomes of Cryptosporidium spp. indicate that two kinds of proteins are involved in adhesion: mucin- like glycoproteins and thrombospondin-related adhesive proteins (TRAPs) ${ }^{38}$.

Glycoprotein 900 (GP900) was the first cloned and characterized mucin-like protein of C. parvum, and it is located at the micronemes of the sporozoites, where it is released to mediate invasion. This protein shares homology with human MUC2 and MUC5 mucins and has several domains: two mucin-like, polythreonine, trinucleotide repeat domains; two cysteine-rich domains; one large degenerate 8-mer amino acid repeat domain; one transmembrane domain; and a signal peptide ${ }^{10}$. The glycoprotein gp900 is involved in parasite attachment and invasion of host cells.

The surface gp60 protein from sporozoites and merozoites is also called gp15/40/60, is a mucinlike molecule and is processed into mature gp40 and gp15 glycopeptides, both having a core of $O$-linked $\alpha$-GalNAc, and gp15 having also a CPI anchor. This protein presents great variability and has been used to classify Cryptosporidium isolates into subtypes ${ }^{36}$.

P23 is a $23-\mathrm{kDa}$ sporozoite and merozoite surface glycoprotein belonging to a family of proteins of 23-27 $\mathrm{kDa}$, each of which has a core of $O$-linked $\alpha$-GalNAc. P23 is antigenically conserved in isolates from different parts of the world and is deposited on trails during the initial stages of infection. This protein is involved in attachment and invasion ${ }^{37}$.

P12 is a glycoprotein detected by phage display and has an $\mathrm{N}$-terminal signal peptide, a transmembrane region, an $\mathrm{N}$-glycosylation site, a casein kinase II phosphorylation site and two N-myristoylation sites; it is located in the apical region of sporozoites and on the surface of oocysts ${ }^{39}$.

A Cryptosporidium parvum protein that contains the C-type lectin domain (CTLD) was described and named $\mathrm{CpClec}^{40}$. It is a type 1 transmembrane protein that contains, in addition to a CTLD, a mucin-like domain predicted to be $O$-glycosylated and a Tyr-based sorting motif in the cytoplasmic tail. The expression of the $\mathrm{CpClec}$ protein is regulated during development, being located in the apical region and in the dense granules of sporozoites and merozoites and in the feeder organelle in intracellular stages, suggesting possible roles in the union, invasion and/or intracellular development in the host cell. In addition, $\mathrm{CpClec}$ mediates the infection of $C$. parvum through $\mathrm{Ca}^{2+}$-dependent binding with sulfated proteoglycans on host intestinal epithelial cells ${ }^{41}$.

$\mathrm{P} 30$ is a lectin in C. parvum and $C$. hominis that specifically binds with the Gal/GalNAc molecules of the host cell. This protein is found in the apical region of sporozoites, is involved in cell binding and invasion and is associated with gp900 and gp40 for this purpose ${ }^{42}$. 
The Cpa135 protein is expressed and secreted by oocysts and sporozoites and, after the invasion of the sporozoites into the host cell, it overlays the parasitophore vacuole. It is a modular protein that has a signal peptide at the $\mathrm{N}$-terminus, a domain similar to ricin B between residues 170 and 290, an LCCL domain between residues 738 and 828 , and a region rich in cysteines at the $\mathrm{C}$-terminus ${ }^{43}$.

The circumsporozoite-like glycoprotein (CSL) is a conserved molecule with a molecular weight of $1,300 \mathrm{kDa}$ that is found in the apical complex of both sporozoites and merozoites and is an important surface ligand that mediates the binding with and facilitates the invasion of intestinal epithelial cells. CSL contains a sporozoite and merozoite ligand that is involved in parasite attachment to intestinal epithelial cells during the infection process in a dose-dependent, saturable, auto-shift manner and, once bound, significantly diminishes the permissiveness of the cell to infection by $C$. parvum sporozoites. The host receptor recognized by CSL was characterized and detected on the microvillar surface of the Caco-2 human intestinal epithelial cell line. It was identified as an $85-\mathrm{kDa}$ surface protein that is capable of significantly inhibiting the binding and invasion of sporozoites that are incubated with it, prior to being inoculated into Caco- 2 cells. Monoclonal antibodies directed to CSL were shown to completely neutralize sporozoite infectivity in vitro and limit an infection in an in vivo mouse model ${ }^{44}$.

The thrombospondin-related adhesive proteins (TRAPs) contain thrombospondin type 1 (TSP1), the genes of which have been conserved among apicomplexans and contain one or more TSP-1 domains, which are implicated in zoite gliding motility and invasion of host cells ${ }^{45}$. TRAP-C1 (Cryptosporidium 1 thrombospondin-related adhesive protein) is a $76-\mathrm{kDa}$ protein located in the apical pole of sporozoites that is structurally related to the micronemal proteins MIC2, TRAP and Etp100, sharing structural homology with members of the thrombospondin adhesive protein family in other apicomplexans ${ }^{46}$. TRAPs and other structurally related proteins constitute a family of functionally homologous proteins involved in parasite motility and cell penetration by binding with sulfated glycosaminoglycans (GAGs) on the host cell surface ${ }^{47}$. It is believed that gliding motility and cell invasion, which depend on the microfilaments of the parasite, result from a capping activity of the parasite surface ligands directly associated with TRAP-C1 molecules ${ }^{48}$.

\section{Blastocystis spp.}

Blastocystis is the most common unicellular parasite that infects the intestinal tract of humans, as well as many animals; it is prevalent worldwide and is estimated to affect between 1 and 2 billion people, although its clinical importance remains controversial ${ }^{49}$.

Multiple morphological forms of Blastocystis can be found in different individuals, such as vacuolar, multivacuolar, avacuolar, granular, ameboid and cystic forms. However, only four stages have been formally accepted: vacuolar, granular, trophozoite and cyst, and Blastocystis also have great genetic diversity ${ }^{50}$.

At first, Blastocystis was named Blastocystis hominis because it was detected mainly in humans; however, it was later determined that there were other hosts for this parasite, and the name was changed to Blastocystis spp. Subsequently, with the use of small-subunit ribosomal RNA gene (SSU-rDNA) analysis, 17 subtypes (STs) were identified, and their presence worldwide has been widely documented. Of the 17 STs, nine (STs 1-9) can infect humans, with STs 1, 2, 3 and 4 being those found in more than $90 \%$ of human isolates ${ }^{51}$. Regarding clinical presentations, Blastocystis carriers infected with ST1 or ST3 had referred intestinal disorders, while ST2 has been associated with asymptomatic infections ${ }^{52}$.

In late $20^{\text {th }}$ century, during a survey focused on determining the surface composition of Blastocystis using lectins, alpha-D-mannose, alpha-D-glucose, N-acetylalpha-D-glucosamine, alpha-L-fucose, chitin and sialic acid were detected ${ }^{53}$.

It has been argued that the ability of Blastocystis to inhibit the host immune response may favor its persistence, adhesion and survival within the intestinal tissue, which can be facilitated by the release of molecules in the hostparasite interface. Cysteine proteases play central roles in the virulence of Blastocystis; for example, the 31-kDa asparaginil cysteine protease called legumain is found on the parasite's cell surface, and cathepsin $\mathrm{B}$, which is $31 \mathrm{kDa}$, represents another virulence factor. It has been observed that legumain plays a pro-survival role, as it induces apoptosis of parasite cells when they react with specific antibodies ${ }^{54}$. These two proteases were identified in parasite culture supernatants and may be involved in increasing intestinal permeability, degradation of human secretory immunoglobulin A, induction of IL-8 production, mucus degradation, tissue damage and gastrointestinal disturbances ${ }^{55}$.

Little is known about the Blastocystis enzymes involved in the degradation of the host's intestinal barriers; however, the parasite genome has been sequenced, and 75 probable proteins have been detected, including proteases, glycosidases, lectins, glycosyl transferases and protease inhibitors, which may facilitate Blastocystis binding to the host. Based on these sequencing data, it was proposed that eight glycosyltransferases and one carbohydrate-binding 


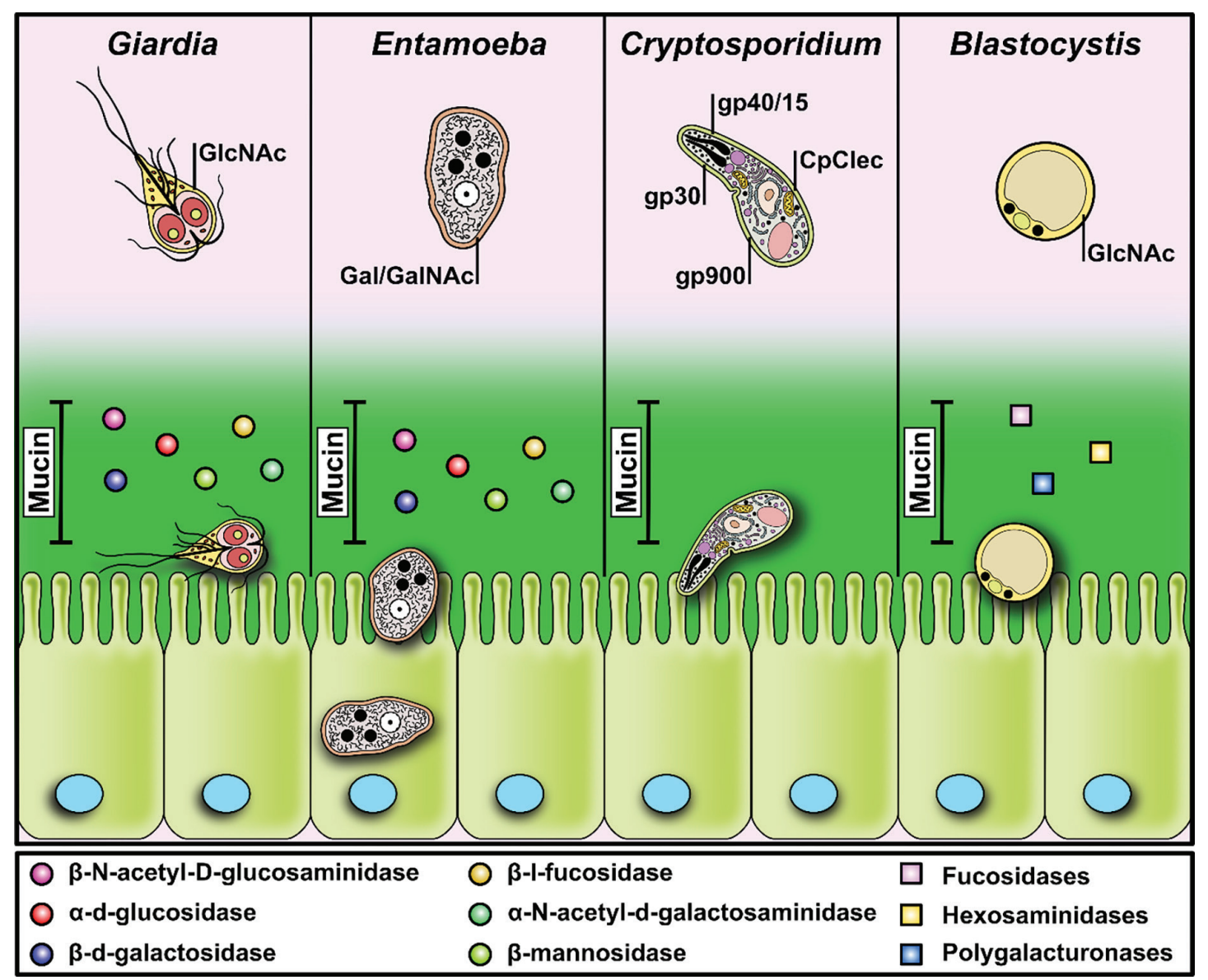

Figure 1 - Schematic representation of the interactions between the main protozoa intestinal pathogens and the human intestinal mucosa. In Giardia, lectin-like structures have been identified on the surface of the parasite, including GIcNAc, mannose and sialic acid as main components that can also be excreted/secreted by the parasite. E. histolytica and Cryptosporidium spp. express specific Gal/GalNAc (named gp30 in cryptosporidium) lectins that facilitate cell adhesion. In addition, Cryptosporidium gp40/15, gp900 and a $\mathrm{CpClec} \mathrm{lectin-like} \mathrm{glycoprotein} \mathrm{have} \mathrm{been} \mathrm{I} \mathrm{described} \mathrm{and} \mathrm{are} \mathrm{involved} \mathrm{in} \mathrm{adhesion} \mathrm{to} \mathrm{intestinal} \mathrm{cells,} \mathrm{forming} \mathrm{an} \mathrm{adhesion-}$ attack complex. G. lamblia and Entamoeba hystolytica can also produce glycosidases such as: $\beta$-N-acetyl-D-glucosaminidase, $\alpha$-d-glucosidase, $\beta$-d-galactosidase, $\beta$-l-fucosidase, $\alpha-\mathrm{N}$-acetyl-d-galactosaminidase and $\beta$-mannosidase. In Blastocystis, $\alpha$-Dmannose, $\alpha$-D-glucose, GlcNAc, $\alpha$-D-Fucose, chitin and sialic acid have been identified on the surface of the parasite. Fucosidases, hexosaminidases and polygalacturonases, which may be involved in the mucin degradation process, have also been described in the predicted Blastocystis secretoma.

protein were secreted. The sequence of several hydrolases has also been determined, and these hydrolases can be used by the parasite for the degradation of host tissues. Among these hydrolases, fucosidase, hexosaminidase and polygalacturonase can degrade host glycoproteins, while proteases can act in several of the processes involved in the invasion and virulence of the parasite ${ }^{56}$.

The function of glycoconjugates found on the surfaces of parasitic protozoa, involves more than serving as a simple additional barrier to the cell membrane; they play important roles in the adhesion and invasion of the parasite and its evasion of the host immune response. The variation in the composition of the surface glycoconjugates of different Blastocyst STs has been determined by electron microscopy, and the modifications observed in the images may be associated with differences in ST pathogenicity ${ }^{57}$.
When phylogeny-based methods and strict validation criteria are used, it was found that approximately $25 \%$ of Blastocystis genes were recently acquired by lateral gene transfer (LGT) from prokaryotic and eukaryotic donors and that this process is ongoing. They reported 167 genes belonging to 74 gene families that corresponded to recent LGT events. The acquired genes come mainly from the bacteria that inhabit the intestine and are central to the adaptation of the colonization in the intestinal tract. Seventeen genes participating in carbohydrate metabolism were identified, including five genes involved in the import and metabolism of L-fucose, such as alpha L-fucosidase (FUCA), L-fucose permease fucP and L-fuculose phosphate aldolase, which are homologous enzymes in Bacteroides thetaiotaomicron, and L-fucose dehydrogenase and L-fuconolactase, and are similar to those found in a metabolic 
pathway in Campylobacter jejuni. On the other hand, at least seven genes with the ability to participate in the evasion of the host's immune response inducing pathogenicity have been described. In particular, a gene encoding a beta-1,3galactosyltransferase $(\beta 1,3 \mathrm{GalT})$ appears to have been acquired from animals and subsequently duplicated several times in the Blastocystis genome. The authors hypothesize that these genes code for proteins that are potentially involved in "molecular mimicry" and serve as camouflage on the cell surface, thereby avoiding recognition by the host $^{58}$. After LGT, extensive duplication of genes is very common and occurs in Blastocystis and in other organisms. Fucose transporters represent one of the families that have undergone a large increase, with seven to nine copies in all STs. These transporters may be relevant for the colonization of the intestine by the parasite, as they are involved in the concentration and regulation of fucose. Despite the advances that have been made in the understanding of the interactions of Blastocystis with the intestinal epithelium and the immunity associated with the intestinal mucosa, there are still many questions to be answered.

\section{CONCLUSION}

The present review focuses on the interactions of the host mucous membrane with lectins expressed on the surface and excreted/secreted and the glycosidases secreted by parasites and their roles during adhesion and colonization of human intestinal mucin cells. However, information on these molecules, particularly glycosidases in parasites, is scarce. In contrast, a number of references about bacterial glycosidases have been published. The studies described herein indicate that the host/parasite interface is dynamic and there is a constant exchange of molecules that can prevent or facilitate host colonization. In addition, the symbiotic coexistence with the intestinal microbiota promotes an adequate microenvironment for the survival of parasites, either by the direct action of the parasites excreted/secreted lectins and glycosidases into the intestinal microenvironment or by the use of hydrolysed carbohydrates as energy sources for their own metabolic pathways. Furthermore, it is necessary to characterize and evaluate more glycosidases, which thus far have only cursorily described the genome in several parasites.

\section{AUTHOR'S CONTRIBUTIONS}

All authors prepared the bibliographic survey, manuscript preparation and approved the final version of the manuscript.

\section{CONFLICT OF INTERESTS}

The authors declare that they have no conflict of interests.

\section{FUNDING}

This work was supported by Consejo Nacional de Ciencia y Tecnología (CONACYT) grant $\mathrm{N}^{\circ}$ 168619. Joel Martínez Ocaña is a doctoral student from Programa de Doctorado em Ciencias Biológicas, Universidad Nacional Autonoma de Mexico

(UNAM).

\section{REFERENCES}

1. Linden SK, Sutton P, Karlsson NG, Korolik V, McGuckin MA. Mucins in the mucosal barrier to infection. Mucosal Immunol. 2008;1:183-97.

2. Theodoropoulos G, Hicks SJ, Corfield AP, Miller BG, Carrington SD. The role of mucins in host-parasite interactions: Part II helminth parasites. Trends Parasitol. 2001;17:130-5.

3. Johansson ME, Sjövall H, Hansson GC. The gastrointestinal mucus system in health and disease. Nat Rev Gastroenterol Hepatol. 2013;10:352-61.

4. Kim YS, Ho SB. Intestinal goblet cells and mucins in health and disease: recent insights and progress. Curr Gastroenterol Rep. 2010;12:319-30.

5. McGuckin MA, Lindén SK, Sutton P, Florin TH. Mucin dynamics and enteric pathogens. Nat Rev Microbiol. 2011;9:265-78.

6. Dhanisha SS, Guruvayoorappan C, Drishya S, Abeesh P. Mucins: structural diversity, biosynthesis, its role in pathogenesis and as possible therapeutic targets. Crit Rev Oncol Hematol. 2018;122:98-122.

7. Singh RS, Bhari R, Kaur HP. Characteristics of yeast lectins and their role in cell-cell interactions. Biotechnol Adv. 2011;29:726-31.

8. Singh RS, Walia AK, Kanwar JR. Protozoa lectins and their role in host-pathogen interactions. Biotechnol Adv. 2016;34:101829.

9. Moody-Haupt S, Patterson JH, Mirelman D, McConville MJ. The major surface antigens of Entamoeba histolytica trophozoites are GPI-anchored proteophosphoglycans. J Mol Biol. 2000;297:409-20.

10. Barnes DA, Bonnin A, Huang JX, Gousset L, Wu J, Gut J, et al. A novel multi-domain mucin-like glycoprotein of Cryptosporidium parvum mediates invasion. Mol Biochem Parasitol. 1998;96:93-110.

11. Cevallos AM, Zhang X, Waldor MK, Jaison S, Zhou X, Tzipori $\mathrm{S}$, et al. Molecular cloning and expression of a gene encoding 
Cryptosporidium parvum glycoproteins gp40 and gp15. Infect Immun. 2000;68:4108-16.

12. Hicks SJ, Theodoropoulos G, Carrington SD, Corfield AP. The role of mucins in host-parasite interactions: Part I - Protozoan parasites. Parasitol Today. 2000;16:476-81.

13. Samuelson J, Robbins P. A simple fibril and lectin model for cyst walls of Entamoeba and perhaps Giardia. Trends Parasitol. 2011;27:17-22.

14. Chatterjee A, Carpentieri A, Ratner DM, Bullitt E, Costello CE, Robbins PW, et al. Giardia cyst wall protein 1 is a lectin that binds to curled fibrils of the GalNAc homopolymer. PLoS Pathog. 2010;19:e1001059.

15. Sousa MC, Gonçalves CA, Bairos VA, Poiares-Da-Silva J. Adherence of Giardia lamblia trophozoites to Int-407 human intestinal cells. Clin Diagn Lab Immunol. 2001;8:258-65.

16. Connaris S, Greenwell P. Glycosidases in mucin-dwelling protozoans. Glycoconj J. 1997;14:879-82.

17. Li E, Tako EA, Singer SM. Complement activation by Giardia duodenalis parasites through the lectin pathway contributes to mast cell responses and parasite control. Infect Immun. 2016;84:1092-9.

18. Jiménez JC, Morelle W, Michalsky JC, Dei-Cas E. Excreted/ secreted glycoproteins of G. intestinalis play an essential role in the antibody response. Parasitol Res. 2007;100:715-20.

19. Chadee K, Johnson ML, Orozco E, Petri WA Jr, Ravdin JI. Binding and internalization of rat colonic mucins by the galactose $/ \mathrm{N}$ acetyl-D-galactosamine adherence lectin of Entamoeba histolytica. J Infect Dis. 1988;158:398-406.

20. Aguirre-García M, Gutiérrez-Kobeh L, López Vancell R. Entamoeba histolytica: adhesins and lectins in the trophozoite surface. Molecules. 2015;20:2802-15.

21. Chadee K, Petri WA Jr, Innes DJ, Ravdin JI. Rat and human colonic mucins bind to and inhibit the adherence lectin of Entamoeba histolytica. J Clin Invest. 1987;80:1245-54.

22. Coudrier E, Amblard F, Zimmer C, Roux P, Olivo-Marin JC, Rigothier MC, et al. Myosin II and the Gal-GalNAc lectin play a crucial role in tissue invasion by Entamoeba histolytica. Cell Microbiol. 2005;7:19-27.

23. Petri WA Jr, Haque R, Mann BJ. The bittersweet interface of parasite and host: lectin-carbohydrate interactions during human invasion by the parasite Entamoeba histolytica. Annu Rev Microbiol. 2002;56:39-64.

24. Katz U, Ankri S, Stolarsky T, Nuchamowitz Y, Mirelman D. Entamoeba histolytica expressing a dominant negative $\mathrm{N}$-truncated light subunit of its gal-lectin are less virulent. Mol Biol Cell. 2002;13:4256-65.

25. Cheng XJ, Hughes MA, Huston CD, Loftus B, Gilchrist CA, Lockhart LA, et al. Intermediate subunit of the Gal/GalNAc lectin of Entamoeba histolytica is a member of a gene family containing multiple CXXC sequence motifs. Infect Immun. 2001;69:5892-8.
26. Min X, Feng M, Guan Y, Man S, Fu Y, Cheng X, et al. Evaluation of the C-terminal fragment of Entamoeba histolytica Gal/GalNAc lectin intermediate subunit as a vaccine candidate against amebic liver abscess. PLoS Negl Trop Dis. 2016;10:e0004419.

27. Galván-Moroyoqui JM, Del Carmen Domínguez-Robles M, Meza I. Pathogenic bacteria prime the induction of Toll-like receptor signalling in human colonic cells by the Gal/GalNAc lectin Carbohydrate Recognition Domain of Entamoeba histolytica. Int J Parasitol. 2011;41:1101-12.

28. Kato K, Makiuchi T, Cheng X, Tachibana H. Comparison of hemolytic activity of the intermediate subunit of Entamoeba histolytica and Entamoeba dispar lectins. PLoS One. 2017;12:e0181864.

29. Ocádiz R, Orozco E, Carrillo E, Quintas LI, Ortega-López J, García-Pérez RM, et al. EhCP112 is an Entamoeba histolytica secreted cysteine protease that may be involved in the parasitevirulence. Cell Microbiol. 2005;7:221-32.

30. Betanzos A, Bañuelos C, Orozco E. Host invasion by pathogenic amoebae: epithelial disruption by parasite proteins. Genes (Basel). 2019;10:618.

31. Nok AJ, Rivera W. Characterization of sialidase from Entamoaeba hystolitica and possible pathogenic role in amebiasis. Parasitol Res. 2003;89:302-7.

32. Espinosa-Cantellano M, Martínez-Palomo A. Pathogenesis of intestinal amebiasis: from molecules to disease. Clin Microbiol Rev. 2000;13:318-31.

33. Thibeaux R, Weber C, Hon CC, Dillies MA, Avé P, Coppée JY, et al. Identification of the virulence landscape essential for Entamoeba histolytica invasion of the human colon. PLoS Pathog. 2013;9:e1003824.

34. Que X, Reed SL. Cysteine proteinases and the pathogenesis of amebiasis. Clin Microbiol Rev. 2000;13:196-206.

35. Gibson AR, Striepen B. Cryptosporidium. Curr Biol. 2018;28:R193-4.

36. Peng MM, Xiao L, Freeman AR, Arrowood MJ, Escalante AA, Weltman AC, et al. Genetic polymorphism among Cryptosporidium parvum isolates: evidence of two distinct human transmission cycles. Emerg Infect Dis. 1997;3:56773.

37. Bouzid M, Hunter PR, Chalmers RM, Tylera KM. Cryptosporidium pathogenicity and virulence. Clin Microbiol Rev. 2013;26:11534.

38. Wanyiri J, Ward H. Molecular basis of Cryptosporidium-host cell interactions: recent advances and future prospects. Future Microbiol. 2006;1:201-8.

39. Yao L, Yin J, Zhang X, Liu Q, Li J, Chen L, et al. Cryptosporidium parvum: identification of a new surface adhesion protein on sporozoite and oocyst by screening of a phage-display cDNA library. Exp Parasitol. 2007;115:333-8.

40. Bhalchandra S, Ludington J, Coppens I, Ward HD. Identification and characterization of Cryptosporidium parvum $\mathrm{Clec}$, a novel 
C-type lectin domain-containing mucin-like glycoprotein. Infect Immun. 2013;81:3356-65.

41. Ludington JG, Ward HD. The Cryptosporidium parvum C-type lectin $\mathrm{CpClec}$ mediates infection of intestinal epithelial cells via interactions with sulfated proteoglycans. Infect Immun. 2016;84:1593-1602.

42. Bhat N, Joe A, PereiraPerrin M, Ward HD. Cryptosporidium p30, a galactose/N-acetylgalactosamine-specific lectin, mediates infection in vitro. J Biol Chem. 2007;282:34877-87.

43. Tosini F, Agnoli A, Mele R, Gomez Morales MA, Pozio E. A new modular protein of Cryptosporidium parvum, with ricin $\mathrm{B}$ and LCCL domains, expressed in the sporozoite invasive stage. Mol Biochem Parasitol. 2004;134:137-47.

44. Langer RC, Schaefer DA, Riggs MW. Characterization of an intestinal epithelial cell receptor recognized by the Cryptosporidium parvum sporozoite ligand CSL. Infect Immun. 2001;69:1661-70.

45. Deng M, Rutherford MS, Abrahamsen MS. Host intestinal epithelial response to Cryptosporidium parvum. Adv Drug Deliv Rev. 2004;56:869-84.

46. Boulter-Bitzer JI, Lee H, Trevors JT. Molecular targets for detection and immunotherapy in Cryptosporidium parvum. Biotechnol Adv. 2007;25:13-44.

47. Naitza S, Spano F, Robson KJ, Crisanti A. The thrombospondinrelated protein family of Apicomplexan parasites: the gears of the cell invasion machinery. Parasitol Today. 1998;14:479-84.

48. Kappe S, Bruderer T, Gantt S, Fujioka H, Nussenzweig V, Ménard R. Conservation of a gliding motility and cell invasion machinery in Apicomplexan parasites. J Cell Biol. 1999;147:937-44.
49. Roberts T, Stark D, Harkness J, Ellis J. Update on the pathogenic potential and treatment options for Blastocystis sp. Gut Pathog. 2014;6:17.

50. Tan KS. New insights on classification, identification, and clinical relevance of Blastocystis spp. Clin Microbiol Rev. 2008;21:639-65.

51. Stensvold CR, Clark CG. Current status of Blastocystis: a personal view. Parasitol Int. 2016;65:763-71.

52. Boorom KF, Smith H, Nimri L, Viscogliosi E, Spanakos G, Parkar U, et al. Oh my aching gut: irritable bowel syndrome, Blastocystis, and asymptomatic infection. Parasit Vectors. 2008;1:40.

53. Lanuza MD, Carbajal JA, Borrás R. Identification of surface coat carbohydrates in Blastocystis hominis by lectin probes. Int $\mathbf{J}$ Parasitol. 1996;26:527-32.

54. Wu B, Yin J, Texier C, Roussel M, Tan KS. Blastocystis legumain is localized on the cell surface, and specific inhibition of its activity implicates a pro-survival role for the enzyme. J Biol Chem. 2010;285:1790-8.

55. Wawrzyniak I, Texier C, Poirier P, Viscogliosi E, Tan KS, Delbac F, El Alaoui H. Characterization of two cysteine proteases secreted by Blastocystis ST7, a human intestinal parasite. Parasitol Int. 2012;61:437-42.

56. Denoeud F, Roussel M, Noel B, Wawrzyniak I, Da Silva C, Diogon $\mathrm{M}$, et al. Genome sequence of the stramenopile Blastocystis, a human anaerobic parasite. Genome Biol. 2011;12:R29.

57. Yason JA, Tan KS. Membrane surface features of Blastocystis subtypes. Genes (Basel). 2018;9:417.

58. Eme L, Gentekaki E, Curtis B, Archibald JM, Roger AJ. Lateral gene transfer in the adaptation of the anaerobic parasite Blastocystis to the gut. Curr Biol. 2017;27:807-20. 